\title{
Ten Problems in Experimental Mathematics
}

\author{
David H. Bailey* Jonathan M. Borwein†, Vishaal Kapoor \\ and Eric Weisstein ${ }^{\S}$
}

September 21, 2004

\section{Introduction}

This article was stimulated by the recent SIAM "100 Digit Challenge" of Nick Trefethen, beautifully described in [12]. Indeed, these ten numeric challenge problems are also listed in [14], where they are followed by the ten symbolic/numeric challenge problems that are discussed in this article. Our intent was to present ten problems that are characteristic of the sorts of problems that commonly arise in "experimental mathematics" $[14,15]$. The challenge in each case is to obtain a high precision numeric evaluation of the quantity, and then, if possible, to obtain a symbolic answer, ideally one with proof. Our goal in this article is to provide solutions to these ten problems, and in the process present a concise account of how one combines symbolic and numeric computation, which may be termed "hybrid computation," in the process of mathematical discovery.

The passage from object, $\alpha$, to answer, $\Omega$, often relies on being able to compute the object to sufficiently high precision, for example to determine numerically whether $\alpha$ is algebraic or is a rational combination of known constants. While some of this is now automated in mathematical computing software such as Maple and Mathematica, in most cases intelligence is needed, for example to choose the search space or to decide the degree of polynomial to hunt for. In a similar sense, using symbolic computing tools such as those incorporated in Maple and Mathematica often requires significant human interaction to produce material results. Such matters are discussed in greater detail in $[14,15]$.

*Lawrence Berkeley National Laboratory, Berkeley, CA 94720. dhbailey@lbl.gov. This work supported by the Director, Office of Computational and Technology Research, Division of Mathematical, Information, and Computational Sciences of the U.S. Department of Energy, under contract number DE-AC03-76SF00098; also by the NSF, under Grant DMS-0342255.

${ }^{\dagger}$ Faculty of Computer Science, Dalhousie University, Halifax, NS, B3H 2 W5. jmborwein@cs.dal.ca. This work supported in part by NSERC and the Canada Research Chair Programme.

${ }^{\ddagger}$ Department of Mathematics, University of BC, Vancouver, BC, V6T 1Z2. vkapoor@math.ubc.ca. This work performed as part of an independent study project.

$\S$ Wolfram Research Inc., Champaign, IL. eww@wolfram.com. 


\section{Integer Relation Detection}

Several of these solutions involve the usage of integer relation detection schemes to experimentally find a likely relationship. Given a real vector $\left(x_{1}, x_{2}, \cdots, x_{n}\right)$, an integer relation algorithm is a computational scheme that either finds the $n$ integers $\left(a_{i}\right)$, not all zero, such that $a_{1} x_{1}+a_{2} x_{2}+\cdots a_{n} x_{n}=0$, or else establishes that there is no such integer vector within a ball of some radius about the origin, where the metric is the Euclidean norm $\left(a_{1}^{2}+a_{2}^{2}+\cdots+a_{n}^{2}\right)^{1 / 2}$.

At the present time, the best known integer relation algorithm is the PSLQ algorithm [24] of Helaman Ferguson, who is well-known in the community for his mathematical sculptures. Simple formulations of the PSLQ algorithm and several variants are given in [7]. Another widely used integer relation detection scheme involves the Lenstra-LenstraLovasz (LLL) algorithm. The PSLQ algorithm, together with related lattice reduction schemes such as LLL, was recently named one of ten "algorithms of the century" by the publication Computing in Science and Engineering [3].

Perhaps the best-known application of PSLQ is the 1995 discovery, by means of a PSLQ computation, of the "BBP" formula for $\pi$ :

$$
\pi=\sum_{k=0}^{\infty} \frac{1}{16^{k}}\left[\frac{4}{8 k+1}-\frac{2}{8 k+4}-\frac{1}{8 k+5}-\frac{1}{8 k+6}\right] .
$$

This formula permits one to directly calculate binary or hexadecimal digits beginning at the $n$-th digit, without needing to calculate any of the first $n-1$ digits [6]. This result has, in turn, led to more recent results that suggest a possible route to a proof that $\pi$ and some other mathematical constants are 2-normal, or in other words that every $m$-long binary string occurs in the binary expansion with limiting frequency $b^{-m}[8,9]$. The BBP formula even has some practical applications: it is used, for example, in the g95 compiler for transcendental function evaluations [33].

All integer relation schemes require very high precision arithmetic, both in the input data and in the operation of the algorithm. Simple reckoning shows that if an integer relation solution vector $\left(a_{i}, 1 \leq i \leq n\right)$ has Euclidean norm $10^{d}$, then the input data must be specified to at least $d n$ digits, or the true solution will be lost in a sea of numerical artifacts. In some cases, including one mentioned at the end of Section 1 below, thousands of digits are required before a solution can be found with these methods. This is the principal reason for the great interest in high-precision numerical evaluations in experimental mathematics research. It is the also the motivation behind this set of ten challenge problems.

\section{The Bifurcation Point $B_{3}$}

\section{Problem}

Compute the value of $r$ for which the chaotic iteration $x_{n+1}=r x_{n}\left(1-x_{n}\right)$, starting with some $x_{0} \in(0,1)$, exhibits a bifurcation between 4-way periodicity and 8-way periodicity. 
Extra credit: This constant is an algebraic number of degree not exceeding 20. Find the minimal polynomial with integer coefficients that it satisfies.

\section{History and Context}

The chaotic iteration $x_{n+1}=r x_{n}\left(1-x_{n}\right)$ has been studied since the early days of chaos theory in the 1950s. It is often called the "logistic iteration," since it mimics the behavior of an ecological population which, if its growth one year outstrips its food supply, often falls back in numbers for the following year, thus continuing to vary in a highly irregular fashion. When $r$ is less than one, iterates of the logistic iteration converge to zero. For $r$ in the range $1<r<B_{1}=3$, iterates converge to some nonzero limit. For $B_{1}<r<B_{2}=1+\sqrt{6}=3.449489 \ldots$, the limiting behavior bifurcates - every other iterate converges to a distinct limit point. For $B_{2}<r<B_{3}$, iterates hop between a set of four distinct limit points; for $B_{3}<r<B_{4}$, iterates hop between a set of eight distinct limit points; and so forth, until $r>B_{\infty}=3.569945672 \ldots$, when the iteration is completely chaotic. See Figure 1. The limiting ratio $\lim _{n}\left(B_{n}-B_{n-1}\right) /\left(B_{n+1}-B_{n}\right)=4.669201 \ldots$ is known as Feigenbaum's delta constant.

A very readable description of the logistic iteration and its role in modern chaos theory are given in Gleick's book [25]. Indeed, John von Neumann had suggested using the logistic map as a random number generator in the late 1940s. Work by W. Ricker in 1954 and detailed analytic studies of logistic maps beginning in the 1950s with Paul Stein and Stanislaw Ulam showed the existence of complicated properties of this type of map beyond simple oscillatory behavior [34, pp. 918-919].

\section{Solution}

We first describe how to obtain a highly accurate numerical value of $B_{3}$ using a relatively straightforward search scheme. Other schemes could be used to find $B_{3}$; we present this one to underscore the fact that computational results sufficient for the purposes of experimental mathematics can often be obtained without resorting to highly sophisticated techniques.

Let $f_{8}(r, x)$ be the eight-times iterated evaluation of $r x(1-x)$, and let $g_{8}(r, x)=$ $f_{8}(r, x)-x$. Imagine a 3-D graph, where $r$ ranges from left to right and $x$ ranges from bottom to top (as in Figure 1), and where $g_{8}(r, x)$ is plotted in the vertical (out-of-plane) dimension. Given some initial $r$ slightly less than $B_{3}$, compute a "comb" of function values at $n$ evenly spaced $x$ values (with spacing $h_{x}$ ) near the limit of the iteration $x_{n+1}=$ $f_{8}\left(r, x_{n}\right)$. In our implementation, we use $n=12$, and we start with $r=3.544, x=$ $0.364, h_{r}=10^{-4}$ and $h_{x}=5 \times 10^{-4}$. With this construction, the comb has $n / 2$ negative function values, followed by $n / 2$ positive function values. Then increment $r$ by $h_{r}$ and reevaluate the "comb," continuing in this fashion until two sign changes are observed among the $n$ function values of the "comb." This means that a bifurcation occurred just prior to the current value of $r$, so restore $r$ to its previous value (by subtracting $h_{r}$ ), reduce $h_{r}$ say by a factor of four, and also reduce the $h_{x}$ say by a factor of 2.5. Continue in this fashion, 


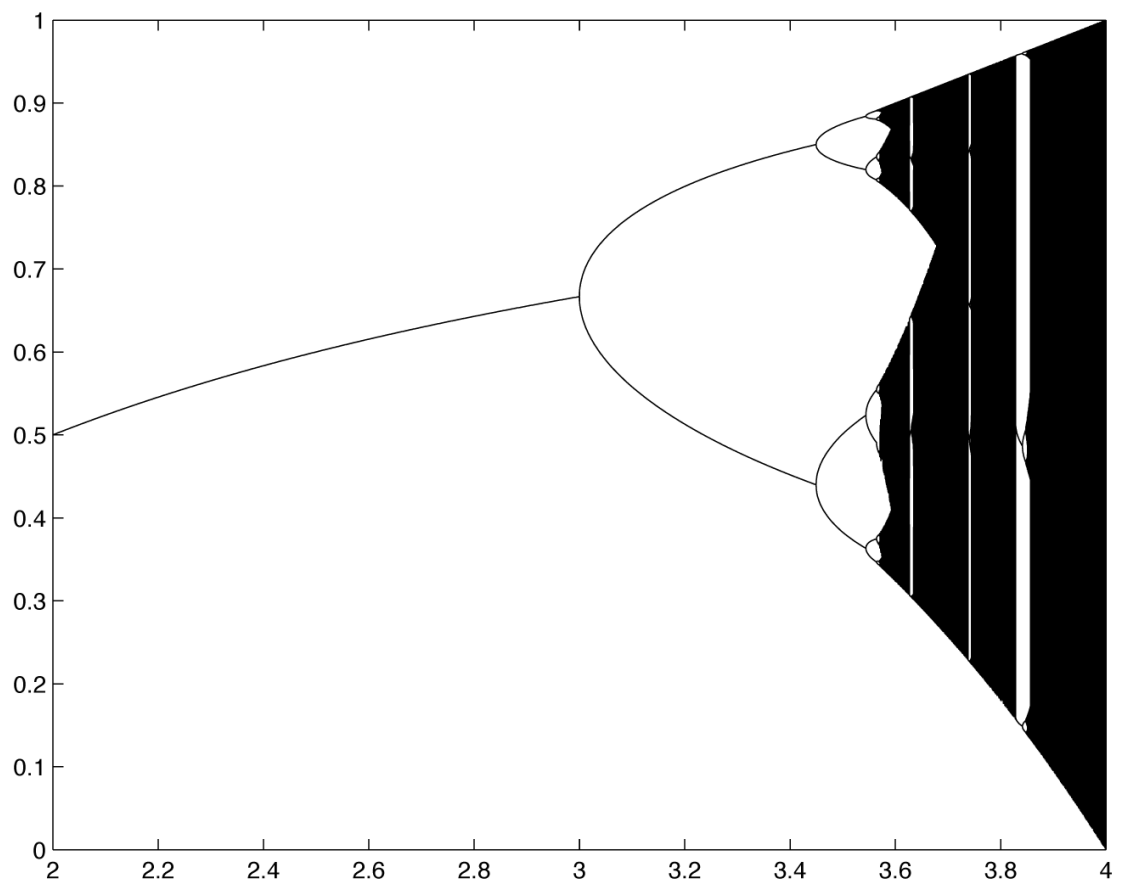

Figure 1: Bifurcation in the logistic iteration.

moving the value of $r$ and its associated "comb" back and forth near the bifurcation point with progressively smaller intervals $h_{r}$. The center of the comb in the $x$ direction must be periodically adjusted to insure that $n / 2$ negative function values are followed by $n / 2$ positive function values, and the spacing parameter $h_{x}$ must be periodically adjusted to insure that two sign changes are disclosed when this occurs. Quit when the smallest of the $n$ function values is within two or three orders of magnitude of the "epsilon" of the arithmetic (e.g., for 2000-digit working precision, "epsilon" is $10^{-2000}$ ). The final value of $r$ is then the desired value $B_{3}$, accurate to within a tolerance given by the final value of $r_{h}$. With 2000-digit working precision, our implementation of this scheme finds $B_{3}$ to 1330-digit accuracy in about five minutes on a 2004-era computer. The first 100 digits are:

$$
\begin{aligned}
B_{3}= & 3.544090359551922853615965986604804540583099845444573675457812530 \\
& 3058429428588630122562585664248917999626 \ldots
\end{aligned}
$$

With even a moderately accurate value of $r$ in hand (at least 200 digits or so), one can use a PSLQ program (such as the PSLQ programs available at the URL

http://crd.lbl.gov/ ${ }^{\sim}$ dhbailey/mpdist) to check to see if $r$ is an algebraic constant. This is done by computing the vector $\left(1, r, r^{2}, \cdots, r^{n}\right)$ for various $n$, beginning with a small value such as 2 or 3 , and then searching for integer relations among these $n+1$ real numbers. When $n \geq 12$, the relation

$$
\begin{aligned}
0= & r^{12}-12 r^{11}+48 r^{10}-40 r^{9}-193 r^{8}+392 r^{7}+44 r^{6}+8 r^{5}-977 r^{4} \\
& -604 r^{3}+2108 r^{2}+4913
\end{aligned}
$$


can be recovered.

A symbolic solution that explicitly produces the polynomial (1) can be obtained as follows. We seek a sequence $x_{1}, x_{2}, . ., x_{4}$ that satisfies the equations

$$
\begin{aligned}
x_{2} & =r x_{1}\left(1-x_{1}\right), x_{3}=r x_{2}\left(1-x_{2}\right), x_{4}=r x_{3}\left(1-x_{3}\right), x_{1}=r x_{4}\left(1-x_{4}\right), \quad \text { and } \\
1 & =\left|\prod_{i=1}^{4} r\left(1-2 x_{i}\right)\right| .
\end{aligned}
$$

The first four conditions represent a period- 4 sequence in the logistic equation $x_{n+1}=$ $r x_{n}\left(1-x_{n}\right)$, and the last condition represents the stability of the cycle which must be \pm 1 for a bifurcation point (see [32] for details).

First, we deal with the system corresponding to $1+\prod_{i=1}^{4} r\left(1-2 x_{i}\right)=0$. We compute the lexicographic Groebner basis in Maple

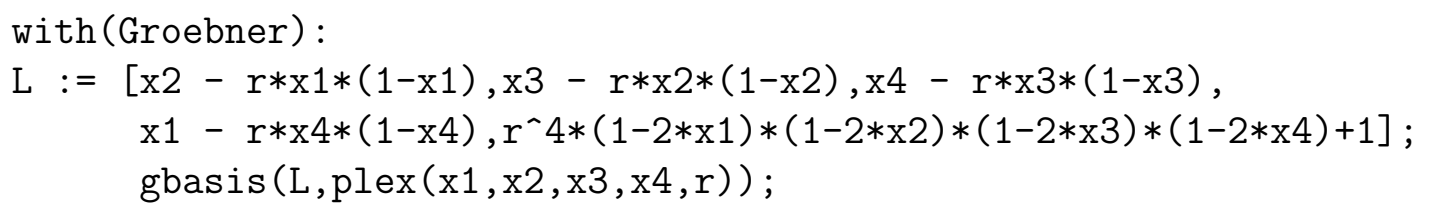

After a cup of coffee, one discovers the univariate element

$$
\begin{aligned}
\left(r^{4}+1\right)\left(r^{4}-8 r^{3}+24 r^{2}-32 r+17\right) & \times\left(r^{4}-4 r^{3}-4 r^{2}+16 r+17\right) \\
\left(r^{12}-12 r^{11}+48 r^{10}-40 r^{9}-193 r^{8}+392 r^{7}\right. & +44 r^{6}+8 r^{5}-977 r^{4} \\
& \left.-604 r^{3}+2108 r^{2}+4913\right)
\end{aligned}
$$

in the Groebner basis - because the monomial ordering is lexicographical with $r$ last.

The first three of these polynomials have no real roots, and the fourth has four real roots. Using trial and error, it is easy to determine that $B_{3}$ is the root of the minimal polynomial

$$
\begin{aligned}
r^{12}-12 r^{11}+48 r^{10}-40 r^{9}-193 r^{8} & +392 r^{7}+44 r^{6}+8 r^{5}-977 r^{4} \\
- & 604 r^{3}+2108 r^{2}+4913,
\end{aligned}
$$

which has the numerical value given above. The corresponding Mathematica code is

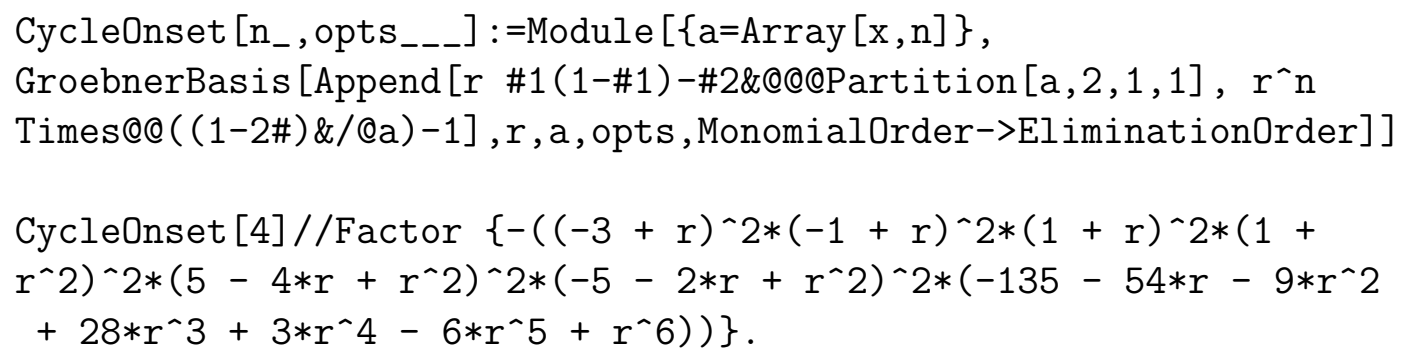

These can be recreated very quickly in Magma, a noncommercial algebraic package available at http://magma.maths.usyd.edu.au/magma: 


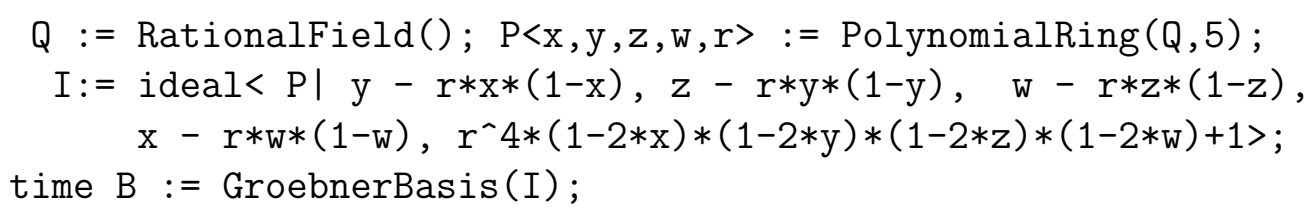

This took 0.050 seconds on a 2.4Ghz Pentium 4. (The Maple computation recovered the polynomial after 930 seconds on a 700Mhz Pentium 3.)

The significantly more challenging problem of computing and analyzing the constant $B_{4}=3.564407266095 \cdots$ is discussed in [7]. In this study, conjectural reasoning suggested that $B_{4}$ might satisfy a 240-degree polynomial, and further that $\alpha=-B_{4}\left(B_{4}-2\right)$ might satisfy a 120 -degree polynomial. The constant $\alpha$ was then computed to over 10,000 digit accuracy, and an advanced three-level multi-pair PSLQ program was employed, running on a parallel computer system, to find an integer relation for the vector $\left(1, \alpha, \alpha^{2}, \cdots, \alpha^{120}\right)$. A numerically significant solution was obtained, with integer coefficients descending monotonically from $257^{30}$, which is a 73 -digit integer, to the final value, which is one (a striking result that is exceedingly unlikely to be a numerical artifact). This experimentally discovered polynomial was recently confirmed in a large symbolic computation [29].

\section{Madelung's Constant}

\section{Problem}

Evaluate

$$
\sum_{(m, n, p) \neq 0} \frac{(-1)^{m+n+p}}{\sqrt{m^{2}+n^{2}+p^{2}}},
$$

where convergence means the limit of sums over the integer lattice points enclosed in increasingly large cubes surrounding the origin.

Extra credit: Usefully identify this constant.

\section{History and Context}

Highly conditionally convergent sums like this are very common in physical chemistry where they are usually written down with no thought of convergence. The sum in question arises as an idealization of the electrochemical stability of $\mathrm{NaCl}$. One computes the total potential at the origin when placing positive and negative charges at each non-zero point of the cubic lattice, [15, Ch. 4].

\section{Solution}

It is important to realize that this sum must be viewed as the limit of the sum in successively larger cubes. The sum diverges when spheres are used instead. To clarify this consider 


$$
b_{2}(s):=\sum_{\substack{m, n \in \mathbb{Z} \\(m, n) \neq 0}} \frac{(-1)^{m+n}}{\left(m^{2}+n^{2}\right)^{s / 2}}, \quad b_{3}(s):=\sum_{\substack{(m, n, p) \in \mathbb{Z} \\(m, n, p) \neq 0}} \frac{(-1)^{m+n+p}}{\left(m^{2}+n^{2}+p^{2}\right)^{s / 2}}
$$

These converges in 2D and 3D over increasing cubes for all Res $>0$. For $s=1$, one may sum over circles in 2D but not in 3D and one may not sum over diamonds in 2D. Many chemists do not know that $b_{3}(1) \neq \sum_{n}(-1)^{n} r_{3}(n) / \sqrt{n}$ which arises by summing over increasing spheres but which diverges. Indeed, the number of representations of $n$ as a sum of three squares, $r_{3}(n)$, is quite irregular - no number of the form $8 n+7$ has such a representation - and is not $O\left(n^{1 / 2}\right)$. This matter is somewhat neglected in the discussion of Madelung's constant in Julian Havil's deservedly popular recent book "Gamma: Exploring Euler's constant," [26], which contains a wealth of information related to each of our problems in which Euler had a hand.

Straightforward methods to compute (6) are extremely unproductive. Such techniques produce at most three digits-and indeed the physical model should have a solar-system sized salt crystal to justify ignoring the boundary. Thus, we are led to using more sophisticated methods. Notate

$$
b_{3}(s)=\sum^{\prime} \frac{(-1)^{i+j+k}}{\left(i^{2}+j^{2}+k^{2}\right)^{s / 2}},
$$

where $\sum^{\prime}$ is a sum over $Z^{3} \backslash\{(0,0,0)\}$, and let $M_{s}(f)$ denote the Mellin transform

$$
M_{s}(f):=\int_{0}^{\infty} f(x) x^{s-1} d x .
$$

The quantity that we wish to compute is $b_{3}(1)$. It follows by symmetry that

$$
\begin{aligned}
b_{3}(1) & =\sum^{\prime} \frac{(-1)^{i+j+k}\left(i^{2}+j^{2}+k^{2}\right)}{\left(i^{2}+j^{2}+k^{2}\right)^{3 / 2}} \\
& =3 \sum^{\prime} \frac{(-1)^{i}\left(i^{2}\right)(-1)^{j+k}}{\left(i^{2}+j^{2}+k^{2}\right)^{3 / 2}} .
\end{aligned}
$$

Note that $M_{s}\left(e^{-t}\right)=\Gamma(s)$ so

$$
M_{3 / 2}\left(q^{n^{2}+j^{2}+k^{2}}\right)=\Gamma\left(\frac{3}{2}\right)\left(n^{2}+j^{2}+k^{2}\right)^{3 / 2}
$$

where $n, j, k$ are arbitrary integers, and $q:=e^{-t}$. Continuing, can rewrite equation (7) as

$$
\begin{aligned}
\Gamma\left(\frac{3}{2}\right) b_{3}(1) & =3 \sum_{n=-\infty}^{\infty} n^{2} M_{3 / 2}\left(\sum(-1)^{j+k} q^{n^{2}+j^{2}+k^{2}}\right) \\
& =3 M_{3 / 2}\left(\sum_{n=-\infty}^{\infty}(-1)^{n} n^{2} q^{n^{2}} \theta_{4}^{2}(x)\right),
\end{aligned}
$$


where $\theta_{4}(x):=\sum_{-\infty}^{\infty}(-1)^{n} x^{n^{2}}$ is the usual Jacobi theta function with $z=0, q=4$. Since $\left.\theta_{4}\left(e^{-\pi / s}\right)=\sqrt{s} \theta_{2}\left(e^{-s \pi}\right)\right)$, it follows that

$$
\Gamma\left(\frac{3}{2}\right) b_{3}(1)=3 \sum_{n=-\infty}^{\infty} n^{2} M_{3 / 2}\left(\sum(-1)^{n} n^{2} q^{n^{2}} \frac{\pi}{x} \theta_{2}^{2}\left(\frac{\pi^{2}}{x}\right)\right) .
$$

Also, $\Gamma\left(\frac{3}{2}\right)=\sqrt{\pi} / 2$, so

$$
b_{3}(1)=12 \sqrt{\pi} \sum_{n=1}^{\infty}(-1)^{n} n^{2} \sum_{\substack{j, k=-\infty \\ j, k \text { odd }}}^{\infty} \int_{0}^{\infty}\left[e^{-n^{2} x-\left(\pi^{2} / 4 x\right)\left(j^{2}+k^{2}\right)}\right] x^{-1 / 2} d x .
$$

The integral is evaluated in exercise 4 of Section 2.2 of [18] and is

$$
\left(\frac{\pi}{n^{2}}\right)^{1 / 2} e^{-\pi n \sqrt{j^{2}+k^{2}}}
$$

SO

$$
b_{3}(1)=48 \pi \sum_{j, k=0}^{\infty} \sum_{n=1}^{\infty}(-1)^{n} n e^{-\pi n \sqrt{\left((2 j+1)^{2}+(2 k+1)^{2}\right.}} .
$$

Finally, for $a>0$,

SO

$$
4 \sum_{n=1}^{\infty}(-1)^{n+1} n e^{-a n}=\frac{4 e^{-a}}{\left(1+e^{-a}\right)^{2}}=\operatorname{sech}^{2}\left(\frac{a}{2}\right),
$$

$$
b_{3}(1)=12 \pi \sum_{\substack{m, n=1, m \\ n \text { odd }}} \operatorname{sech}^{2}\left(\frac{\pi}{2}\left(m^{2}+n^{2}\right)^{1 / 2}\right) .
$$

Summing for $m, n \leq 81$ in the previous equation gives us

$$
\begin{aligned}
b_{3}(1)= & 1.74756459463318219063621203554439740348516143662474175 \\
& 8152825350765040623532761179890758362694607891 \ldots
\end{aligned}
$$

It is possible to accelerate the convergence further still. Details can be found in $[18,15]$.

There are closed forms for sums with an even number of variables, up to 24 and beyond. For example $b_{2}(2 s)=-4 \alpha(s) \beta(s)$ where $\alpha(s):=\sum_{n \geq 0}(-1)^{n} /(n+1)^{s}$ and $\beta(s):=\sum_{n \geq 0}(-1)^{n} /(2 n+1)^{s}$. In particular, $b_{2}(2)=-\pi \log 2$. No such closed form for $b_{3}$ is known, while much work has been put into looking for one. The formula for $b_{2}$ is due to Lorenz (1879). It was rediscovered by G.H. Hardy and is equivalent to Jacobi's Lambert series formula for $\theta_{3}^{2}(q)$

$$
\theta_{3}^{2}(q)-1=4 \sum_{n \geq 0}(-1)^{n} \frac{q^{2 n+1}}{1-q^{2 n+1}} .
$$

This in turn is equivalent to the formula for the number of representations of $n$ as a sum of two squares, counting order and sign,

$$
r_{2}(n)=4\left(d_{1}(n)-d_{3}(n)\right)
$$

where $d_{k}$ is the number of divisors of $n$ congruent to $k$ modulo four. The analysis of three squares is notoriously harder. 


\section{Double Euler Sums}

\section{Problem}

Evaluate the sum

$$
\sum_{k=1}^{\infty}\left(1-\frac{1}{2}+\cdots+(-1)^{k+1} \frac{1}{k}\right)^{2}(k+1)^{-3}
$$

Extra credit: Evaluate this constant as a multiterm expression involving well-known mathematical constants. This expression has seven terms, and involves $\pi, \log 2, \zeta(3)$, and $\operatorname{Li}_{5}(1 / 2)$, where the polylogarithm $\operatorname{Li}_{n}(x)=\sum_{k>0} x^{n} / n^{k}$.

Hint: The expression is "homogenous," in the sense that each term has the same total "degree." The degrees of $\pi$ and $\log 2$ are each 1 , the degree of $\zeta(3)$ is 3 , the degree of $\operatorname{Li}_{5}(1 / 2)$ is 5, and the degree of $\alpha^{n}$ is $n$ times the degree of $\alpha$.

\section{History and Context}

In April 1993, Enrico Au-Yeung, an undergraduate at the University of Waterloo, brought to the attention of one of us (Borwein) the curious result

$$
\sum_{k=1}^{\infty}\left(1+\frac{1}{2}+\cdots+\frac{1}{k}\right)^{2} k^{-2}=4.59987 \ldots \approx \frac{17}{4} \zeta(4)=\frac{17 \pi^{4}}{360} .
$$

The function $\zeta(s)$ in (18) is the classical Riemann zeta function,

$$
\zeta(s)=\sum_{n=1}^{\infty} \frac{1}{n^{s}}
$$

Euler had solved Bernoulli's Basel problem when he showed that for even integers, $\zeta(2 n)$ is an explicit rational multiple of $\pi^{2 n}$, [15, Section 3.2].

$\mathrm{Au}$-Yeung had computed the sum in (18) to 500,000 terms, giving an accuracy of 5 or 6 decimal digits. Suspecting that his discovery was merely a modest numerical coincidence, Borwein sought to compute the sum to a higher level of precision. Using Fourier analysis and Parseval's equation, he obtained

$$
\frac{1}{2 \pi} \int_{0}^{\pi}(\pi-t)^{2} \log ^{2}\left(2 \sin \frac{t}{2}\right) d t=\sum_{n=1}^{\infty} \frac{\left(\sum_{k=1}^{n} \frac{1}{k}\right)^{2}}{(n+1)^{2}}
$$

The idea here is that the series on the right of (19) permits one to evaluate (18), while the integral on the left can be computed using the numerical quadrature facility of Mathematica or Maple. When he did this, he was surprised to find that the conjectured identity holds to more than 30 digits. We should add here that by good fortune, 17/360 = $0.047222 \ldots$ has period one and thus can plausibly be recognized from its first six digits, so that Au-Yeung's numerical discovery was not entirely far-fetched. 


\section{Solution}

First define the multi-valued zeta function

$$
\zeta\left(s_{1}, s_{2}, \cdots, s_{k}\right):=\sum_{n_{1}>n_{2}>\cdots>n_{k}>0} \prod_{j=1}^{k} n_{j}^{-\left|s_{j}\right|} \sigma_{j}^{-n_{j}},
$$

where the $s_{1}, s_{2}, \ldots, s_{k}$ are non-zero integers, and the $\sigma_{j}:=\operatorname{signum}\left(\mathrm{s}_{\mathrm{j}}\right)$. A fast method for computing such sums based on Hölder Convolution is discussed in [19] and implemented in the EZFace + interface, which is available as an online tool at the URL

http://www.cecm.sfu.ca/projects/ezface+. Expanding the squared term in (17), we have

$$
\sum_{\substack{0<i, j<k \\ k>0}} \frac{(-1)^{i+j+1}}{i j k^{3}}=-2 \zeta(3,-1,-1)+\zeta(3,2) .
$$

Evaluating this in EZFace+ we quickly obtain

$$
\begin{aligned}
C= & 0.156166933381176915881035909687988193685776709840303872957529354 \\
& 497075037440295791455205653709358147578 \ldots
\end{aligned}
$$

Given this numerical value, PSLQ or some other integer-relation-finding tool can be used to see if this constant satisfies a rational linear relation with the following constants (as suggested in the hint above): $\pi^{5}, \pi^{4} \log (2), \pi^{3} \log ^{2}(2), \pi^{2} \log ^{3}(2), \pi \log ^{4}(2), \log ^{5}(2)$, $\pi^{2} \zeta(3), \pi \log (2) \zeta(3), \log ^{2}(2) \zeta(3), \zeta(5), \operatorname{Li}_{5}(1 / 2)$. The result is quickly found to be:

$$
\begin{aligned}
C= & 4 \operatorname{Li}_{5}\left(\frac{1}{2}\right)-\frac{1}{30} \log ^{5}(2)-\frac{17}{32} \zeta(5)-\frac{11}{720} \pi^{4} \log (2)+\frac{7}{4} \zeta(3) \log ^{2}(2) \\
& +\frac{1}{18} \pi^{2} \log ^{3}(2)-\frac{1}{8} \pi^{2} \zeta(3) .
\end{aligned}
$$

This result has been proven in various ways, both analytic and algebraic. Indeed, all evaluations of sums of the form $\zeta\left( \pm a_{1}, \pm a_{2}, \cdots, \pm a_{m}\right)$ with weight $w:=\sum_{k} a_{m}$, for $k<8$, as in (20) are established.

\section{Further History and Context}

What Borwein did not know at the time was that Au-Yeung's suspected identity follows directly from a related result proved by De Doelder in 1991. In fact, it had cropped up even earlier as a problem in the American Mathematical Monthly, but the story goes back further still. Some historical research showed that Euler considered these summations. In response to a letter from Goldbach, he examined sums that are equivalent to

$$
\sum_{k=1}^{\infty}\left(1+\frac{1}{2^{m}}+\cdots+\frac{1}{k^{m}}\right)(k+1)^{-n} .
$$

The great Swiss mathematician was able to give explicit values for certain of these sums in terms of the Riemann zeta function. 
Starting from where we left off in the previous section provides some insight into evaluating related sums. Recall, the Taylor expansion of $f(x):=-\frac{1}{2} \log (1-x) \log (1+x)$ is

$$
\sum_{k=0}^{\infty}\left(1-\frac{1}{2}+\frac{1}{3}-\cdots+\frac{1}{2 k-1}\right) \frac{x^{2 k}}{2 k}
$$

Using Parseval's equation for $f\left(e^{i t}\right)$, we have an effective way of computing

$$
\sum_{k=0}^{\infty} \frac{\left(1-\frac{1}{2}+\frac{1}{3}-\cdots+\frac{1}{2 k-1}\right)^{2}}{(2 k)^{2}}
$$

in terms of an integral which can be rapidly evaluated in Maple or Mathematica.

Alternatively, we may compute

$$
\sum_{k=0}^{\infty} \frac{\left(1+\frac{1}{2}+\frac{1}{3}+\cdots+\frac{1}{k}\right)^{2}}{k^{2}} .
$$

The Fourier expansions of $(\pi-t) / 2$ and $-\log |2 \sin (t / 2)|$ are

$$
\begin{aligned}
& \sum_{n=1}^{\infty} \frac{\sin (n t)}{n}=\frac{\pi-t}{2}, \\
& \sum_{n=1}^{\infty} \frac{\cos (n t)}{n}=-\log |2 \sin (t / 2)|, \text { for all } t \in(0,2 \pi) .
\end{aligned}
$$

Multiplying these together, simplifying, and doing a partial fraction decomposition gives

$$
-\log |2 \sin (t / 2)| \cdot \frac{\pi-t}{2}=\sum_{n=1}^{\infty} \frac{1}{n} \sum_{k=1}^{n-1} \frac{1}{k} \sin (n t) \quad \text { on }(0,2 \pi) .
$$

Applying Parseval's equation gives

$$
\frac{1}{4 \pi} \int_{0}^{2 \pi}(\pi-t)^{2} \log ^{2}(2 \sin (t / 2)) d t=\sum_{n=1}^{\infty} \frac{\left(1+\frac{1}{2}+\frac{1}{3}+\cdots+\frac{1}{n}\right)^{2}}{(n+1)^{2}} .
$$

The integral may be computed numerically in Maple or Mathematica giving an approximation to the sum.

The Clausen functions defined by

$$
\mathrm{Cl}_{2}(\theta):=\sum_{n=1}^{\infty} \frac{\sin (n \theta)}{n^{2}}, \quad \mathrm{Cl}_{3}(\theta):=\sum_{n=1}^{\infty} \frac{\cos (n \theta)}{n^{3}}, \quad \mathrm{Cl}_{4}(\theta):=\sum_{n=1}^{\infty} \frac{\sin (n \theta)}{n^{4}}, \cdots
$$

arise as repeated antiderivatives of (26). They are useful throughout harmonic analysis and elsewhere.

For example, with $\alpha:=2 \arctan \sqrt{7}$, one discovers by PSLQ that

$$
6 \mathrm{Cl}_{2}(\alpha)-6 \mathrm{Cl}_{2}(2 \alpha)+2 \mathrm{Cl}_{2}(3 \alpha) \stackrel{?}{=} 7 \mathrm{Cl}_{2}\left(\frac{2 \pi}{7}\right)+7 \mathrm{Cl}_{2}\left(\frac{4 \pi}{7}\right)-7 \mathrm{Cl}_{2}\left(\frac{6 \pi}{7}\right)
$$


or, in what can be shown to be equivalent,

$$
\frac{24}{7 \sqrt{7}} \int_{\pi / 3}^{\pi / 2} \log \left(\left|\frac{\tan (t)+\sqrt{7}}{\tan (t)-\sqrt{7}}\right|\right) d t \stackrel{?}{=} L_{-7}(2)=1.151925470 \ldots
$$

This arises from the volume of an ideal tetrahedron in hyperbolic space, [14, pp. 90-91]. [Here $L_{-7}(s):=\sum_{n>0} \chi_{-7}(n) n^{-s}$ is the primitive L-series mod seven, whose character pattern is $1,1,-1,1,-1,-1,0$, which is given by $\chi_{-7}(k)=2(\sin (k \tau)+\sin (2 k \tau)-\sin (3 k \tau)) / \sqrt{7}$ with $\tau:=2 \pi / 7$.]

Although (30) has been checked to 2000 decimal digits, using a numerical integration scheme we shall describe in Section 8, and although it is known by K-theoretic reasons that the LHS/RHS of (29) is rational [13], to our knowledge there is no proof of either (29) or (30). We might add that recently two additional conjectured identities related to (30) have been discovered by PSLQ computations. Let $I_{n}$ be the definite integral of (30), except with limits $n \pi / 24$ and $(n+1) \pi / 24$. Then

$$
\begin{array}{ccc}
-2 I_{2}-2 I_{3}-2 I_{4}-2 I_{5}+I_{8}+I_{9}-I_{10}-I_{11} & \stackrel{?}{=} & 0 \\
I_{2}+3 I_{3}+3 I_{4}+3 I_{5}+2 I_{6}+2 I_{7}-3 I_{8}-I_{9} & \stackrel{?}{=} & 0
\end{array}
$$

Readers who attempt to calculate numerical values for either the integral in (30) or the integral $I_{9}$ in (31) should note that the integrand function has a nasty singularity at $t=\arctan \sqrt{7}$.

In retrospect, perhaps it was for the better that Borwein had not known of De Doelder's and Euler's results, because Au-Yeung's intriguing numerical discovery launched a fruitful line of research by a number of researchers that has continued until the present day. Sums of this general form are nowadays known as "Euler sums" or "Euler-Zagier sums." Euler sums may be studied through a profusion of methods: combinatorial, analytic and algebraic. The reader is referred to [15, Ch. 3] for an overview of Euler sums and their applications. We take up the story again in Problem 9.

\section{Khintchine's Constant}

\section{Problem}

Evaluate

$$
K_{0}:=\prod_{k=1}^{\infty}\left[1+\frac{1}{k(k+2)}\right]^{\log _{2} k}=\prod_{k=1}^{\infty} k^{\left[\log _{2}\left(1+\frac{1}{k(k+2)}\right)\right]} .
$$

Extra credit: Evaluate this constant in terms of a less-well-known mathematical constant. 


\section{History and Context}

Consider the experiment of taking a random continued fraction expansion, $\alpha=\left[a_{0}, a_{1}, \cdots\right]$, and forming the limit

$$
K_{0}(\alpha):=\lim _{n \rightarrow \infty}\left(a_{0} a_{1} \cdots a_{n}\right)^{1 / n}
$$

Based on the Gauss-Kuzmin distribution, which establishes that the digit distribution of a random continued fraction satisfies Prob $(a[k]=n)=\log _{2}(1+1 / k /(k+2))$, Khintchine showed that the above limit exists for all continued fractions and that the expected value of the limit is $K_{0}$. This circle of ideas is accessibly developed in [26]. As such a constant has an interesting interpretation, computation seems like the next step.

Taking the log of both sides and simplifying (32), we have

$$
\log 2 \log K_{0}=\sum_{k=1}^{\infty} \log k \log \left(1+\frac{1}{k(k+2)}\right) .
$$

Such a formula converges extremely slowly. Computing the sum of the first 10000 terms gives 2 digits of $\log 2 \log K_{0}$. Thus, direct computation again proves to be quite difficult.

\section{Solution}

Rewrite $\log n$ as the telescoping sum

$$
\log n=(\log n-\log n-1)+\cdots+(\log 2-\log 1)=\sum_{k=2}^{n} \log \left(\frac{k}{k-1}\right) .
$$

Then we see that

$$
\log 2 \log K_{0}=\sum_{n=1}^{\infty} \sum_{k=2}^{\infty} \log (k) \log \frac{(n+1)^{2}}{n(n+2)} .
$$

Interchanging the order of the summation, we obtain

$$
\log 2 \log K_{0}=\sum_{k=2}^{\infty} \sum_{n=1}^{k} \log (k) \log \frac{(n+1)^{2}}{n(n+2)}=\sum_{k=2}^{\infty} \log (k) \sum_{n=k}^{\infty} \log \frac{(n+1)^{2}}{n(n+2)}
$$

But

$$
\sum_{n=k}^{\infty} \log \frac{(n+1)^{2}}{n(n+2)}=\log \frac{(k+1)^{2}}{k(k+2)},
$$

so (37) transforms into

$$
\log 2 \log K_{0}=\sum_{k=2}^{\infty} \log (k) \log \frac{(k+1)^{2}}{k(k+2)}=-\sum_{k=2}^{\infty} \log \left(1-\frac{1}{n}\right) \log \left(1+\frac{1}{n}\right) .
$$


The Taylor series for $-\log (1-x) \log (1+x)$ is

$$
\sum_{k=1}^{\infty}\left(1-\frac{1}{2}+\frac{1}{3}-\cdots+\frac{1}{2 k-1}\right) \frac{x^{2 k}}{k} .
$$

This allows us to rewrite $\log 2 \log K_{0}$ as

$$
\begin{aligned}
\log 2 \log K_{0} & =\sum_{k=1}^{\infty}\left(1-\frac{1}{2}+\frac{1}{3}-\cdots+\frac{1}{2 k-1}\right) \frac{1}{k} \sum_{n=2}^{\infty} n^{-2 k} \\
& =\sum_{k=1}^{\infty}\left(1-\frac{1}{2}+\frac{1}{3}-\cdots+\frac{1}{2 k-1}\right) \frac{1}{k}(\zeta(2 k)-1) .
\end{aligned}
$$

In Maple or Mathematica, we can easily compute this sum. Taking the first 161 terms, we obtain 100 digits of $K_{0}$

$$
\begin{aligned}
K_{0}= & 2.68545200106530644530971483548179569382038229399446295 \\
& 3051152345557218859537152002801141174931847709 \ldots
\end{aligned}
$$

However, faster convergence is still possible and the constant has been computed to more than 7000 places. Moreover, the harmonic and other averages are similarly treated. It appears to satisfy its own predicted behavior; for details, see [5, 31]. Correspondingly, using $10^{8}$ terms $K_{0}(\pi)=2.675 \cdots$ while $K_{0}(e)=\infty=\lim _{n} \sqrt[3 n]{(2 n) !}$. Note that $e=$ $[2,1,2,1,1,4,1,1,6,1,1,8,1,1,10, \cdots]$.

\section{Ramanujan's AGM Continued Fraction}

\section{Problem}

Given $a, b, \eta>0$, define

$$
R_{\eta}(a, b)=\frac{a}{\eta+\frac{b^{2}}{\eta+\frac{4 a^{2}}{\eta+\frac{9 b^{2}}{\eta+\ddots} .}} .}
$$

Calculate $R_{1}(2,2)$.

Extra credit: Evaluate this constant as a two-term expression involving a well-known mathematical constant. 


\section{History and Context}

This continued fraction arises in Ramanujan's Notebooks. He discovered the beautiful fact that

$$
\frac{R_{\eta}(a, b)+R_{\eta}(b, a)}{2}=R_{\eta}\left(\frac{a+b}{2}, \sqrt{a b}\right) .
$$

The authors wished to record this in [14], and wished to computationally check the identity. A first attempt to numerically compute $R_{1}(1,1)$ directly failed miserably, and with some effort only three reliable digits were obtained: $0.693 \cdots$. With hindsight, the slowest convergence of the fraction occurs in the mathematically simplest case, namely when $a=b$. Indeed $R_{1}(1,1)=\log 2$ as the first primitive numerics had tantalizingly suggested.

\section{Solution}

Attempting a direct computation of $R_{1}(2,2)$ using a depth of 20000 gives us two digits. Thus we must seek more sophisticated methods. From (1.11.70) of [15] we see that for $0<b<a$,

$$
\mathcal{R}_{1}(a, b)=\frac{\pi}{2} \sum_{n \in Z} \frac{a K(k)}{K^{2}(k)+a^{2} n^{2} \pi^{2}} \operatorname{sech}\left(n \pi \frac{K\left(k^{\prime}\right)}{K(k)}\right),
$$

where $k=b / a=\theta_{2}^{2} / \theta_{3}^{2}, k^{\prime}=\sqrt{1-k^{2}}$. Here $\theta_{2}, \theta_{3}$ are Jacobian theta functions and $K$ is a complete elliptic integral of the first kind.

Writing the previous equation as a Riemann sum, we have

$$
\mathcal{R}(a):=\mathcal{R}_{1}(a, a)=\int_{0}^{\infty} \frac{\operatorname{sech}(\pi x /(2 a))}{1+x^{2}} d x=2 a \sum_{k=1}^{\infty} \frac{(-1)^{k+1}}{1+(2 k-1) a},
$$

where the final equality follows from the Cauchy-Lindelof Theorem. This sum may also be written as $\mathcal{R}(a)=\frac{2 a}{1+a} F\left(\frac{1}{2 a}+\frac{1}{2}, 1 ; \frac{1}{2 a}+\frac{3}{2} ;-1\right)$. The latter form is what we use in Maple or Mathematica to determine

$$
\begin{aligned}
\mathcal{R}(2)= & 0.97499098879872209671990033452921084400592021999471060574526825 \\
& 1285877387455708594352325320911129362 \ldots
\end{aligned}
$$

This constant, as written, is a bit difficult to recognize, but if one first divides by $\sqrt{2}$, one can obtain, using the Inverse Symbolic Calculator, an online tool available at the URL http://www.cecm.sfu.ca/projects/ISC/ISCmain.html, that the quotient is $\pi / 2-\log (1+$ $\sqrt{2}$ ). Thus we conclude, experimentally, that

$$
\mathcal{R}(2)=\sqrt{2}[\pi / 2-\log (1+\sqrt{2})] .
$$

Indeed, it follows, see [17], that

$$
\mathcal{R}(a)=2 \int_{0}^{1} \frac{t^{1 / a}}{1+t^{2}} d t .
$$


Note that $\mathcal{R}(1)=\log 2$. No non-trivial closed form is known for $\mathcal{R}(a, b)$ with $a \neq b$, although

$$
\mathcal{R}_{1}\left(\frac{1}{4 \pi} \beta\left(\frac{1}{4} \frac{1}{4}\right), \frac{\sqrt{2}}{8 \pi} \beta\left(\frac{1}{4} \frac{1}{4}\right)\right)=\frac{1}{2} \sum_{n \in \mathbf{Z}} \frac{\operatorname{sech}(n \pi)}{1+n^{2}},
$$

is close to closed. It would be pleasant to find a direct proof of (44). Further details are to be found in $[17,16,15]$.

\section{Expected Distance on a Unit Square}

\section{Problem}

Calculate the expected distance between two random points on different sides of the unit square.

$$
E_{2}:=\frac{2}{3} \int_{0}^{1} \int_{0}^{1} \sqrt{x^{2}+y^{2}} d x d y+\frac{1}{3} \int_{0}^{1} \int_{0}^{1} \sqrt{1+(u-v)^{2}} d u d v .
$$

Extra credit: Express this constant as a three-term expression involving algebraic constants and an evaluation of the natural logarithm with an algebraic argument.

\section{History and Context}

This evaluation and the next were discovered, in slightly more complicated form James D. Klein [15, pg 66]. He computed the numerical integral and for comfort compared it to a Monte Carlo simulation. Indeed, a straightforward approach to obtaining a quick numerical value for an iterated integral is to use a Monte-Carlo simulation, which means to approximate the integral as a sum of function values taken at pseudorandomly generated points within the region. It is important to use a good pseudorandom number generator for this purpose. We tried doing a Monte Carlo evaluation for this problem, using a pseudorandom number generator based on the recently discovered class of provably normal numbers [9],[14, pg. 169-70]. The results we obtained for the two integrals above, with $10^{8}$ pseudorandom pairs, are $0.765203 \ldots$ and $1.076643 \ldots$, respectively, yielding an expected distance of $0.869017 \ldots$... Unfortunately, none of these three values immediately suggests a closed form, and they are not sufficiently accurate (because of statistical limitations) to be suitable for PSLQ or other constant recognition tools. More digits are needed.

\section{Solution}

It is possible to calculate high-precision numerical values for these two integrals using a $2-\mathrm{D}$ quadrature (numerical integration) program. In our program, we employed a twodimensional version of the "tanh-sinh" quadrature algorithm, which we will discuss in more detail in Problem 8. Two-dimensional quadrature is usually much more expensive 
than one-dimensional quadrature, at a given precision level, because many more function evaluations must be performed. Often a highly parallel computer system must be used to obtain a high-precision result in reasonable run time [11]. Nonetheless, in this case we were able to evaluate the first of the two integrals to 108-digit accuracy in 21 minutes runtime on a 2004-era computer, and the second to 118-digit accuracy in just 20 seconds. The first is more difficult due to non-differentiability at the origin.

Indeed, in this case both Maple and Mathematica are able to evaluate these integrals, as is, to over 100 decimal digit accuracy in just a few minutes run time. This is because these software packages are able to symbolically integrate the inner integrals, leaving only the outer integrals to be evaluated numerically. Maple, Mathematica, and the 2-D quadrature program all agreed on the following numerical value for the expected distance:

$$
\begin{aligned}
\alpha= & 0.86900905527453446388497059434540662485671927963168056 \\
& 9660350864584179822174693053113213554875435754 \ldots
\end{aligned}
$$

Using PSLQ, with the basis elements $\alpha, \sqrt{2}, \log (\sqrt{2}+1)$ and 1 , we obtain

$$
\alpha=\frac{1}{9} \sqrt{2}+\frac{5}{9} \log (\sqrt{2}+1)+\frac{2}{9} .
$$

An alternate solution is try to evaluate the integrals symbolically! In fact, in this case Version 5.1 of Mathematica can both do the integrals "out of the box", while in the first case Maple appears to need to be coaxed, for instance by converting to polar coordinates:

$$
2 \int_{0}^{\pi / 4} \int_{0}^{\sec \theta} r^{2} d r d \theta=\frac{2}{3} \int_{0}^{\pi / 4} \sec ^{3} \theta d \theta=\frac{1}{3} \sqrt{2}-\frac{1}{6} \log (2)+\frac{1}{3} \log (2+\sqrt{2}),
$$

since the radius for a given $\theta$ is $1 / \cos (\theta)$.

As for the second integral, Maple and Mathematica both give

$$
-\frac{1}{3} \sqrt{2}-\frac{1}{2} \log (\sqrt{2}-1)+\frac{1}{2} \log (1+\sqrt{2})+\frac{2}{3} .
$$

To obtain the second integral analytically, write it as $2 \int_{0}^{1} \int_{0}^{u} \sqrt{1+(u-v)^{2}} d v d u$. Now change variables, $t:=u-v$, to obtain $1 / 2 \int_{0}^{1}\left\{u \sqrt{1+u^{2}}+\operatorname{arcsinh}(u)\right\} d u$.

Thus, the expected distance is

$$
\frac{1}{9} \sqrt{2}-\frac{1}{9} \log (2)+\frac{2}{9} \log (2+\sqrt{2})-\frac{1}{6} \log (\sqrt{2}-1)+\frac{1}{6} \log (1+\sqrt{2})+\frac{2}{9},
$$

which can be simplified to the formula (46) above.

\section{Expected Distance on a Unit Cube}

\section{Problem}

Calculate the expected distance between two random points on different faces of the unit cube. Hint: This can be expressed in terms of integrals as

$$
E_{3}:=\frac{4}{5} \int_{0}^{1} \int_{0}^{1} \int_{0}^{1} \int_{0}^{1} \sqrt{x^{2}+y^{2}+(z-w)^{2}} d w d x d y d z
$$




$$
+\frac{1}{5} \int_{0}^{1} \int_{0}^{1} \int_{0}^{1} \int_{0}^{1} \sqrt{1+(y-u)^{2}+(z-w)^{2}} d u d w d y d z .
$$

Extra credit: Express this constant as a six-term expression involving algebraic constants and two evaluations of the natural logarithm with algebraic arguments.

\section{History and Context}

As we noted earlier, this evaluation was discovered, in essentially the same form, by Klein [15, pg 66]. As with Problem 6, a Monte Carlo integration scheme can be used to obtain a quick approximation to the integral. The values we obtained were $0.870792 \ldots$ and $1.148859 \ldots$, respectively, yielding an expected distance of $0.926406 \ldots$. Once again, however, these numerical values do not immediately suggest a closed-form evaluation, yet the accuracy is too low to apply PSLQ or other constant recognition schemes. What's more, in this case, unlike Problem 6, neither Maple or Mathematica are able to evaluate these four-fold integrals directly - though Mathematica comes close.

As in most cases "help" is needed, in the form of mathematical manipulation to render these integrals into a form where mathematical computing software can evaluate themnumerically or symbolically.

\section{Solution}

One may show that the first integral is

$$
\frac{\sqrt{2 \pi}}{5} \sum_{n=2}^{\infty} \frac{{ }_{2} \mathrm{~F}_{1}(1 / 2,-n+2 ; 3 / 2 ; 1 / 2)}{(2 n+1) \Gamma(n+2) \Gamma(5 / 2-n)}+\frac{4}{15} \sqrt{2}+\frac{2}{5} \log (\sqrt{2}+1)-\frac{1}{75} \pi
$$

and the second integral is

$$
\begin{aligned}
& \frac{\sqrt{\pi}}{10} \sum_{n=0}^{\infty} \frac{{ }_{4} \mathrm{~F}_{3}(1,1 / 2,-1 / 2-n,-n-1 ; 2,1 / 2-n, 3 / 2 ;-1)}{(2 n+1) \Gamma(n+2) \Gamma(3 / 2-n)} \\
& -\frac{2}{25}+\frac{\sqrt{2}}{50}+\frac{1}{10} \log (\sqrt{2}+1) .
\end{aligned}
$$

This is a consequence of using the binomial theorem in each case after integrating with respect to $\mathrm{z}$ in the first case. These expansions allows one to numerically compute the expectation to high precision and to express both of the individual integrals in terms of the same set of constants.

The numerical value of this expectation is

0.926390055174046729218163586547779014444960190107335046732521921271418 $504594036683829313473075349968212 \ldots$

An integer relation search in the span of $\{1, \pi, \sqrt{2}, \sqrt{3}, \log (1+\sqrt{2}), \log (2+\sqrt{3})\}$ produces

$$
\frac{4}{75}+\frac{17}{75} \sqrt{2}-\frac{2}{25} \sqrt{3}-\frac{7}{75} \pi+\frac{7}{25} \log (1+\sqrt{2})+\frac{7}{25} \log (7+4 \sqrt{3}) .
$$


With substantial effort we were able to nurse the symbolic integral out of Maple. We started as in the last problem by integrating of $0<w<z$, and doubling etc., ultimately producing

$$
\begin{aligned}
E_{3}: & =-\int_{0}^{1}\left(2 x^{3}+6 x^{2}+3\right) \ln \left(\sqrt{2+x^{2}}-1\right) d x \\
& +\int_{0}^{1} 3 \frac{-\left(x^{2}+1\right) \ln \left(\sqrt{2+x^{2}-1}\right)+\ln (\sqrt{2}-1)}{x^{2}\left(x^{2}+1\right)} d x \\
& =-\frac{5}{3} \pi+\frac{7}{6} \sqrt{2}+\frac{7}{2} \ln (1+\sqrt{2})-\frac{3}{2} \ln (2)+\ln (1+\sqrt{3})+\frac{37}{24}+\frac{3}{4} \ln (1+\sqrt{2}) \pi,
\end{aligned}
$$

which we leave to the reader to complete.

Mathematica was more helpful: consider

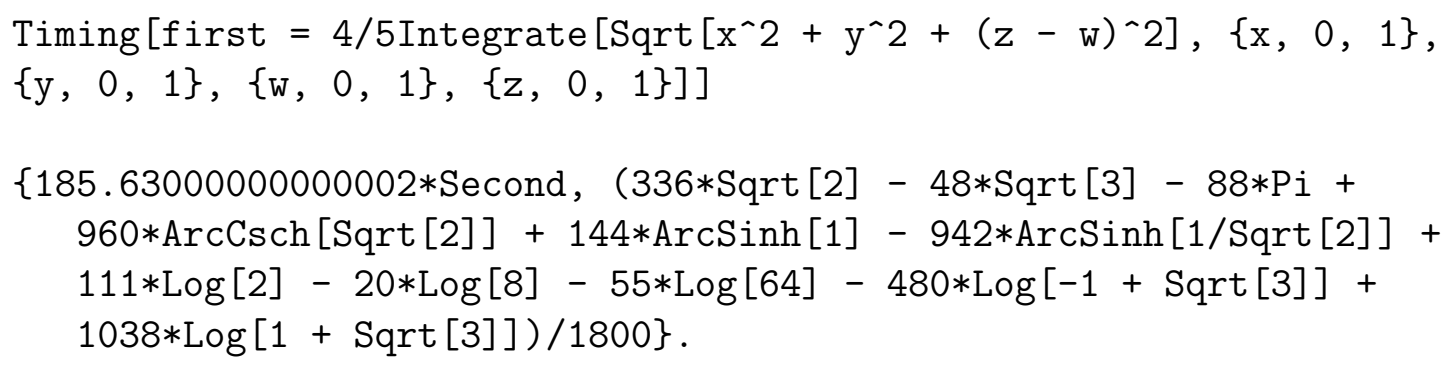

This evaluates the first integral while the second one can be done a little help. The combined result then can be symbolically simplified to the result shown. Nonetheless we need to emphasize that (i) one needs to be confident to try since it took several minutes and (ii) phrases like Maple could not or Mathematica can are highly user and release specific. Hence our desire to show various paths here and elsewhere.

\section{An Infinite Cosine Product}

\section{Problem}

Calculate

$$
\pi_{2}:=\int_{0}^{\infty} \cos (2 x) \prod_{n=1}^{\infty} \cos \left(\frac{x}{n}\right) d x .
$$

\section{History and Context}

The challenge of showing that the value of $\pi_{2}<\pi / 8$ was posed by Bernard Mares, Jr., along with the problem of showing

$$
\pi_{1}:=\int_{0}^{\infty} \prod_{n=1}^{\infty} \cos \left(\frac{x}{n}\right) d x<\frac{\pi}{4} .
$$

This is indeed true, although the error is remarkably small, as we shall see. 


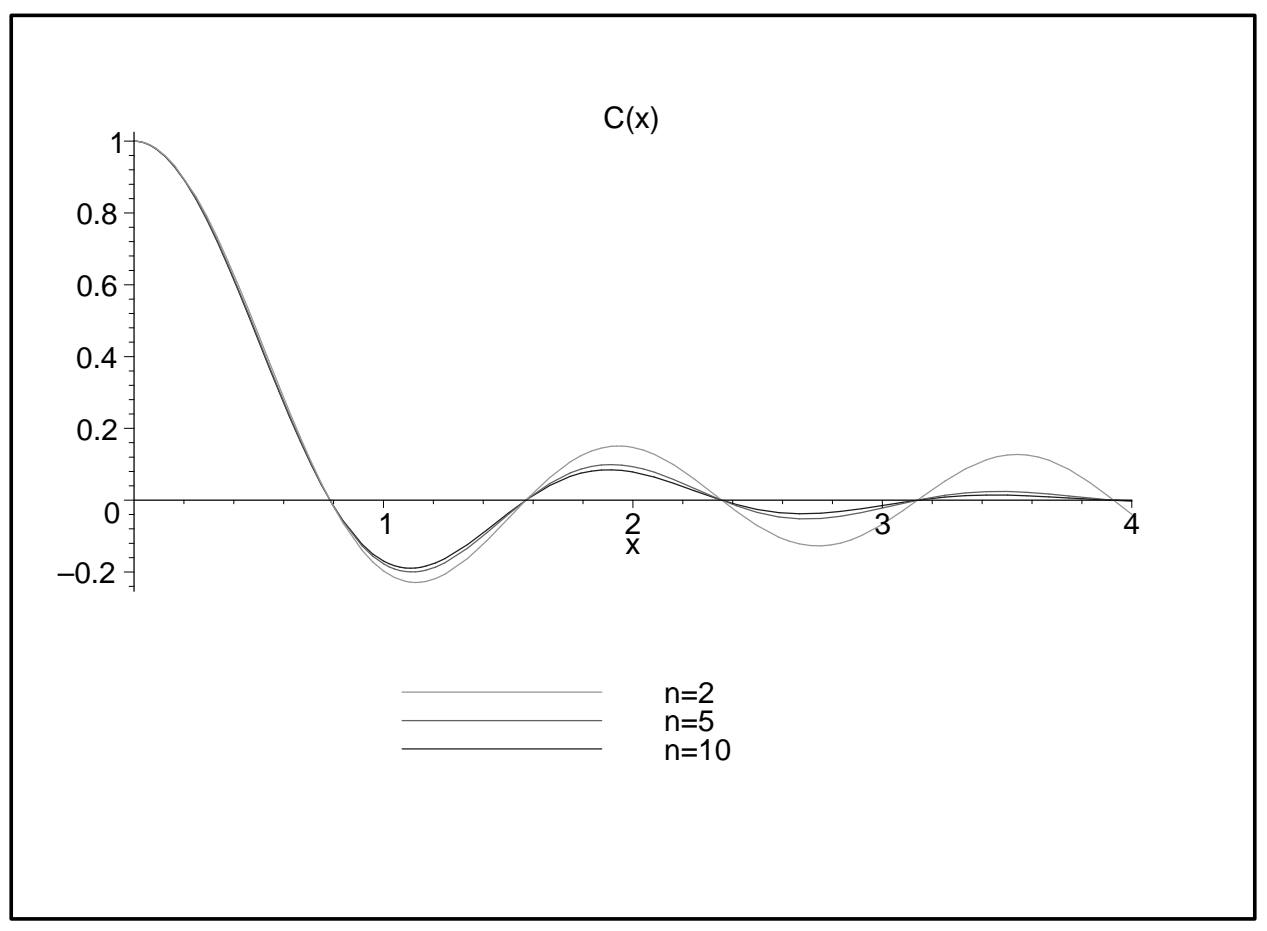

Figure 2: Approximations to $\prod_{n \geq 1} \cos (x / n)$

\section{Solution}

The computation of a high-precision numerical value for this integral is rather challenging, due in part to the oscillatory behavior of $\prod_{n \geq 1} \cos (x / n)$ (see Figure 2), but mostly due to the difficulty of computing high-precision evaluations of the integrand function. Note that evaluating thousands of terms of the infinite product would produce only a few correct digits. Thus it is necessary to rewrite the integrand function in a form more suitable for computation.

Let $f(x)$ be the integrand function. We can write

$$
f(x)=\cos (2 x)\left[\prod_{1}^{m} \cos (x / k)\right] \exp \left(f_{m}(x)\right)
$$

where we choose $m>x$, and where

$$
f_{m}(x)=\sum_{k=m+1}^{\infty} \log \cos \left(\frac{x}{k}\right)
$$

The log cos evaluation can be expanded in a Taylor series [1, pg 75], as follows:

$$
\log \cos \left(\frac{x}{k}\right)=\sum_{j=1}^{\infty} \frac{(-1)^{j} 2^{2 j-1}\left(2^{2 j}-1\right) B_{2 j}}{j(2 j) !}\left(\frac{x}{k}\right)^{2 j},
$$


where $B_{2 j}$ are Bernoulli numbers. Note that since $k>m>x$ in (54), this series converges. We can now write

$$
f_{m}(x)=\sum_{k=m+1}^{\infty} \sum_{j=1}^{\infty} \frac{(-1)^{j} 2^{2 j-1}\left(2^{2 j}-1\right) B_{2 j}}{j(2 j) !}\left(\frac{x}{k}\right)^{2 j}
$$

which after applying the identity [1, pg 807]

$$
B_{2 j}=\frac{(-1)^{j+1} 2(2 j) ! \zeta(2 j)}{(2 \pi)^{2 j}}
$$

and interchanging the sums, we obtain

$$
f_{m}(x)=-\sum_{j=1}^{\infty} \frac{\left(2^{2 j}-1\right) \zeta(2 j)}{j \pi^{2 j}}\left[\sum_{k=m+1}^{\infty} \frac{1}{k^{2 j}}\right] x^{2 j} .
$$

Note that the inner sum can also be written in terms of the zeta function, as follows:

$$
f_{m}(x)=-\sum_{j=1}^{\infty} \frac{\left(2^{2 j}-1\right) \zeta(2 j)}{j \pi^{2 j}}\left[\zeta(2 j)-\sum_{k=1}^{m} \frac{1}{k^{2 j}}\right] x^{2 j}
$$

This can now be written in a compact form for computation as

$$
f_{m}(x)=-\sum_{j=1}^{\infty} a_{j} b_{j, m} x^{2 j}
$$

where

$$
\begin{aligned}
a_{j} & =\frac{\left(2^{2 j}-1\right) \zeta(2 j)}{j \pi^{2 j}} \\
b_{j, m} & =\zeta(2 j)-\sum_{k=1}^{m} 1 / k^{2 j} .
\end{aligned}
$$

Note that $\zeta(2 j), a_{j}$ and $b_{j, m}$ can all be pre-computed, say for $j$ up to some limit, and for a variety of $m$. In our program, which computes this integral to 120 digits accuracy, we pre-compute $b_{j, m}$ for $m=1,2,4,8,16, \ldots, 256$ and for $j$ up to 300. During quadrature computation, the function evaluation program picks $m$ to be the first power of two greater than the argument $x$, and then applies formulas (53) and (56). It is not necessary to compute $f(x)$ for $x>200$, since for these large arguments $|f(x)|<10^{-120}$ and thus may be presumed to be zero.

The computation of the Riemann zeta function values may be done using a simple algorithm due to Peter Borwein [20], or, since what we require is the entire set of values $(\zeta(2 j), 1 \leq j \leq n)$ for some $n$, by a convolution scheme described in [5]. It is important to note that the computation of both the zeta values and also $b_{j, m}$ must be done with a much higher working precision (in our program, we use 1600-digit precision) than the 120 digits required for the quadrature results, since the two terms being subtracted in formula 
(58) are very nearly equal, and these values need to be calculated to a relative precision of 120 digits.

With this evaluation scheme for $f(x)$ in hand, the integral (51) can be computed using, for instance, the tanh-sinh quadrature algorithm, which can be implemented fairly easily on a personal computer or workstation, and which is also well-suited for highly parallel processing $[10,11][15, \mathrm{pg} 312]$. This algorithm approximates an integral $f(x)$ on $[-1,1]$ by transforming it to an integral on $(-\infty, \infty)$, using the change of variable $x=g(t)$, where $g(t)=\tanh (\pi / 2 \cdot \sinh t)$ :

$$
\int_{-1}^{1} f(x) d x=\int_{-\infty}^{\infty} f(g(t)) g^{\prime}(t) d t=h \sum_{j=-\infty}^{\infty} w_{j} f\left(x_{j}\right)+E(h) .
$$

Here $x_{j}=g(h j)$ and $w_{j}=g^{\prime}(h j)$ are abscissas and weights for the tanh-sinh quadrature scheme (which can be pre-computed), and $E(h)$ is the error in this approximation.

Note that $g^{\prime}(t)=\pi / 2 \cdot \cosh t \cdot \operatorname{sech}^{2}(\pi / 2 \cdot \sinh t)$ and its derivatives tend to zero very rapidly for large $|t|$. Thus even if the function $f(t)$ has a vertical derivative, blow-up discontinuity or oscillatory behavior at an endpoint, the product function $f(g(t)) g^{\prime}(t)$ is in many cases quite well-behaved, going rapidly to zero, together with all of its derivatives, for large $|t|$. In such cases, the Euler-Maclaurin summation formula [2, pg. 180] can be used to conclude that the error $E(h)$ in the approximation (59) decreases very rapidlyfaster than any power of $h$. In many applications, the tanh-sinh algorithm achieves quadratic convergence - reducing the interval $h$ in half produces twice as many correct digits in the result.

The tanh-sinh quadrature algorithm is designed for a finite integration interval. In this problem, where the interval of integration is $[0, \infty)$, it is necessary to convert the integral to a problem on a finite interval. This can be done with the simple substitution $s=1 /(x+1)$, which yields an integral from 0 to 1 .

In spite of the substantial computation required to construct the zeta and $b$ arrays, as well as the abscissas $x_{j}$ and weights $w_{j}$ needed for tanh-sinh quadrature, the entire calculation requires only about one minute on a 2004-era computer, using the ARPREC arbitrary precision software package available at http://crd.lbl.gov/ ${ }^{\sim}$ hbailey/mpdist. The first 100 digits of the result are the following:

$$
\begin{aligned}
& 0.392699081698724154807830422909937860524645434187231595926812285162 \\
& 093247139938546179016512747455366777 \ldots
\end{aligned}
$$

Using the Inverse Symbolic Calculator, for instance, one finds that this is likely to be $\pi / 8$. But a careful comparison with a high-precision value of $\pi / 8$, namely

$$
\begin{aligned}
& 0.392699081698724154807830422909937860524646174921888227621868074038 \\
& 477050785776124828504353167764633497 \ldots,
\end{aligned}
$$

reveals that they are not equal - the two values differ by approximately $7.407 \times 10^{-43}$. Indeed, these two values are provably distinct. The reason is governed by the fact that $\sum_{n=1}^{55} 1 /(2 n+1)>2>\sum_{n=1}^{54} 1 /(2 n+1)$. See [15, Ch. 2] for additional details. We do not know a concise closed-form evaluation of this constant. 


\section{Further History and Context}

Recall the sinc function

$$
\operatorname{sinc}(x):=\frac{\sin (x)}{x} .
$$

Consider, the seven highly oscillatory integrals below.

$$
\begin{aligned}
I_{1} & :=\int_{0}^{\infty} \operatorname{sinc}(x) d x=\frac{\pi}{2}, \\
I_{2} & :=\int_{0}^{\infty} \operatorname{sinc}(x) \operatorname{sinc}\left(\frac{x}{3}\right) d x=\frac{\pi}{2} \\
I_{3} & :=\int_{0}^{\infty} \operatorname{sinc}(x) \operatorname{sinc}\left(\frac{x}{3}\right) \operatorname{sinc}\left(\frac{x}{5}\right) d x=\frac{\pi}{2}, \\
\ddots & :=\int_{0}^{\infty} \operatorname{sinc}(x) \operatorname{sinc}\left(\frac{x}{3}\right) \cdots \operatorname{sinc}\left(\frac{x}{11}\right) d x=\frac{\pi}{2} \\
I_{6} & :=\int_{0}^{\infty} \operatorname{sinc}(x) \operatorname{sinc}\left(\frac{x}{3}\right) \cdots \operatorname{sinc}\left(\frac{x}{13}\right) d x=\frac{\pi}{2}
\end{aligned}
$$

However,

$$
\begin{aligned}
I_{8} & :=\int_{0}^{\infty} \operatorname{sinc}(x) \operatorname{sinc}\left(\frac{x}{3}\right) \cdots \operatorname{sinc}\left(\frac{x}{15}\right) d x \\
& =\frac{467807924713440738696537864469}{935615849440640907310521750000} \pi \approx 0.499999999992646 \pi .
\end{aligned}
$$

When this was first found by a researcher, using a well-known computer algebra package, both he and the software vendor concluded there was a "bug" in the software. Not so! It is easy to see that the limit of these integrals is $2 \pi_{1}$. Our analysis, via Parseval's theorem, links the integral

$$
I_{N}:=\int_{0}^{\infty} \operatorname{sinc}\left(a_{1} x\right) \operatorname{sinc}\left(a_{2} x\right) \cdots \operatorname{sinc}\left(a_{N} x\right) d x
$$

with the volume of the polyhedron $P_{N}$ given by

$$
P_{N}:=\left\{x:\left|\sum_{k=2}^{N} a_{k} x_{k}\right| \leq a_{1},\left|x_{k}\right| \leq 1,2 \leq k \leq N\right\}
$$

where $x:=\left(x_{2}, x_{3}, \cdots, x_{N}\right)$.

If we let

$$
C_{N}:=\left\{\left(x_{2}, x_{3}, \cdots, x_{N}\right):-1 \leq x_{k} \leq 1,2 \leq k \leq N\right\},
$$

then

$$
I_{N}=\frac{\pi}{2 a_{1}} \frac{\operatorname{Vol}\left(P_{N}\right)}{\operatorname{Vol}\left(C_{N}\right)}
$$


Thus, the value drops precisely when the constraint $\sum_{k=2}^{N} a_{k} x_{k} \leq a_{1}$ becomes active and bites the hypercube $C_{N}$. That occurs when $\sum_{k=2}^{N} a_{k}>a_{1}$. In the above, $\frac{1}{3}+\frac{1}{5}+\cdots+$ $\frac{1}{13}<1$, but on addition of the term $\frac{1}{15}$, the sum exceeds 1 , the volume drops, and $I_{N}=\frac{\pi}{2}$ no longer holds. A similar analysis applies to $\pi_{2}$. Moreover, it is fortunate that we began with $\pi_{1}$ or the falsehood of the identity analogous to that displayed above would have been much harder to see.

\section{A Multi-Valued Zeta Function}

\section{Problem}

Calculate

$$
\sum_{i>j>k>l>0} \frac{1}{i^{3} j k^{3} l} .
$$

Extra credit: Express this constant as a single-term expression involving a well-known mathematical constant.

\section{History and Context}

We continue the discussion from Problem 4. In the notation introduced there, we ask for the value of $\zeta(3,1,3,1)$. The study of such sums in two variables, as we noted, originates with Euler. These investigations were apparently due to a serendipitous mistake. Euler wrote to Goldbach:

When I recently considered further the indicated sums of the last two series in my previous letter, I realized immediately that the same series arose due to a mere writing error, from which indeed the saying goes, "Had one not erred, one would have achieved less." (Si non errasset, fecerat ille minus).

Euler's reduction formula is

$$
\zeta(s, 1)=\frac{s}{2} \zeta(s+1)-\frac{1}{2} \sum_{k=1}^{s-2} \zeta(k+1) \zeta(s+1-k)
$$

which reduces the given double Euler sums to a sum of products of classical $\zeta$-values.

Euler also noted the first reflection formulae

$$
\zeta(a, b)+\zeta(b, a)=\zeta(a) \zeta(b)-\zeta(a+b),
$$

certainly valid for $a, b>1$. This is an easy algebraic consequence of adding the double sums.

Another marvellous fact is the sum formula

$$
\sum_{\Sigma a_{i}=n, a_{i} \geq 0} \zeta\left(a_{1}+2, a_{2}+1, \cdots, a_{r}+1\right)=\zeta(n+r+1)
$$


for all integer $n, r \geq 0$. This, as David Bradley observes, is equivalent to the generating function identity

$$
\sum_{n>0} \frac{1}{n^{r}(n-x)}=\sum_{k_{1}>k_{2}>\cdots k_{r}>0} \prod_{j=1}^{r} \frac{1}{k_{j}-x} .
$$

The first three non-trivial cases of $(63)$ are $\zeta(3)=\zeta(2,1), \zeta(4)=\zeta(3,1)+\zeta(2,2)$ and $\zeta(2,1,1)=\zeta(4)$

\section{Solution}

We notice that such a function is a generalization of the zeta function. Similar to the definition in Section 4, we define

$$
\zeta\left(s_{1}, s_{2}, \cdots, s_{k} ; x\right)=\sum_{n_{1}>n_{2}>\cdots>n_{k}>0} \frac{x_{1}^{n}}{n_{1}^{s_{1}} n_{2}^{s_{2}} \cdots n_{r}^{s_{r}}},
$$

for $s_{1}, s_{2}, \ldots, s_{k}$ non-negative integers. We see that we are asked to compute $\zeta(3,1,3,1 ; 1)$. Such a sum can be evaluated directly using the EZFace+ interface at http://www.cecm.sfu.ca/projects/ezface+, which employs the Hölder Convolution, giving us the numerical value

$$
\begin{aligned}
& 0.005229569563530960100930652283899231589890420784634635522547448 \\
& 97214886954466015007497545432485610401627 \ldots
\end{aligned}
$$

Alternatively, we may proceed using differential equations. It is easy to see that

$$
\begin{aligned}
\frac{d}{d x} \zeta\left(n_{1}, n_{2}, \cdots, n_{r} ; x\right) & =\frac{1}{x} \zeta\left(n_{1}-1, n_{2}, \cdots, n_{r} ; x\right), \text { for } \mathrm{n}_{1}>1, \text { and } \\
\frac{d}{d x} \zeta\left(n_{1}, n_{2}, \cdots, n_{r} ; x\right) & =\frac{1}{1-x} \zeta\left(n_{2}, \cdots, n_{r} ; x\right), \text { for } \mathrm{n}_{1}=1,
\end{aligned}
$$

with initial conditions $\zeta\left(n_{1} ; 0\right)=\zeta\left(n_{1}, n_{2} ; 0\right)=\cdots=\zeta\left(n_{1}, \cdots, n_{r} ; 0\right)=0$, and $\zeta(; x) \equiv 1$. Solving

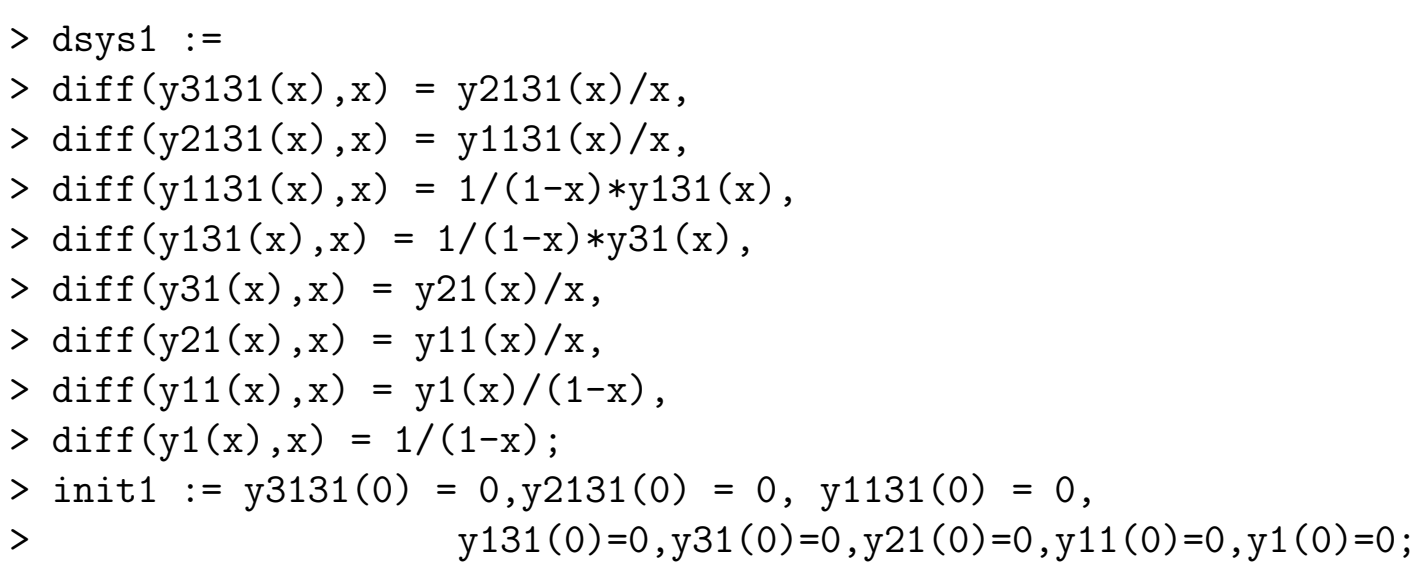


in Maple, we are able to obtain 0.005229569563518039612830536519667669502942 ((this is valid to 13 decimal places). Maple's identify command is unable to identify portions of this number, and the inverse symbolic calculator does not return a result. It should be mentioned that both Maple and the ISC identified the constant $\zeta(3,1)$ (see the History and Context). From the hint for this question, we know this is a single-term expression. Suspecting a similar form to $\zeta(3,1)$, we search for a constants $c, d$ such that $\zeta(3,1,3,1)=$ $c \pi^{d}$ - this leads to $c=1 / 81440=2 / 10$ ! and $d=8$.

\section{Further History and Context}

We start with the simpler value, $\zeta(3,1)$. Notice that

$$
-\log (1-x)=x+\frac{1}{2} x^{2}+\frac{1}{3} x^{3}+\cdots
$$

SO

$$
\begin{aligned}
f(x) & :=-\log (1-x) /(1-x)=x+\left(1+\frac{1}{2}\right) x^{2}+\left(1+\frac{1}{2}+\frac{1}{3}\right) x^{3}+\cdots \\
& =\sum_{n \geq m>0} \frac{x^{n}}{m} .
\end{aligned}
$$

As noted in the Section on double Euler sums above,

$$
\frac{(-1)^{m+1}}{\Gamma(m)} \int_{0}^{1} \log ^{m-1}(x) x^{n} d x=\frac{1}{(n+1)^{m}},
$$

so integrating $f$ using the above transform for $m=3$, we obtain

$$
\begin{aligned}
\zeta(3,1) & =\frac{(-1)}{2} \int_{0}^{1} \log ^{2}(x) f(x) d x \\
& =.270580808427784547879000924 \ldots
\end{aligned}
$$

The corresponding recondite generating function is

$$
\left.\sum_{n \geq 0} \zeta\left(\{3,1\}_{n}\right)\right) x^{4 n}=\frac{\cosh (\pi x)-\cos (\pi x)}{\pi^{2} x^{2}},
$$

equivalent to Zagier's conjectured identity

$$
\zeta\left(\{3,1\}_{n}\right)=\frac{2 \pi^{4 n}}{(4 n+2)} .
$$

Here $\{3,1\}_{n}$ denotes $n$-fold concatenation of $\{3,1\}$.

The proof of this identity (see [15, pg 160]) devolves from a remarkable factorization of the generating function in terms of hypergeometric functions:

$$
\sum_{n \geq 0} \zeta\left(\{3,1\}_{n}\right) x^{4 n}={ }_{2} \mathrm{~F}_{1}\left(x \frac{(1+i)}{2},-x \frac{(1+i)}{2} ; 1 ; 1\right){ }_{2} \mathrm{~F}_{1}\left(x \frac{(1-i)}{2},-x \frac{(1-i)}{2} ; 1 ; 1\right) .
$$


Finally, it can be shown in various ways that

$$
\zeta\left(\{3\}_{n}\right)=\zeta\left(\{2,1\}_{n}\right)
$$

for all $n$, while a proof of

$$
\zeta\left(\{2,1\}_{n}\right) \stackrel{?}{=} 2^{3 n} \zeta\left(\{-2,1\}_{n}\right)
$$

remains elusive.

Only the first case of (72), namely

$$
\sum_{n=1}^{\infty} \frac{1}{n^{2}} \sum_{m=1}^{n-1} \frac{1}{m}=8 \sum_{n=1}^{\infty} \frac{(-1)^{n}}{n^{2}} \sum_{m=1}^{n-1} \frac{1}{m} \quad(=\zeta(3))
$$

has a self-contained proof, [15]. Indeed, the only other proven case is

$$
\sum_{n=1}^{\infty} \frac{1}{n^{2}} \sum_{m=1}^{n-1} \frac{1}{m} \sum_{p=1}^{m-1} \frac{1}{p^{2}} \sum_{q=1}^{p-1} \frac{1}{q}=64 \sum_{n=1}^{\infty} \frac{(-1)^{n}}{n^{2}} \sum_{m=1}^{n-1} \frac{1}{m} \sum_{p=1}^{m-1} \frac{(-1)^{p}}{p^{2}} \sum_{q=1}^{p-1} \frac{1}{q} \quad(=\zeta(3,3)) .
$$

This is an outcome of a complete set of equations for MZV's of depth four.

There has been abundant evidence amassed to support identity (72) since it was found in 1996. For example, very recently Petr Lisonek checked the first 85 cases to 1000 places in about 41 hours with only the expected roundoff error. And he checked $\mathrm{N}=163$ in ten hours. This is the only identification of its type of an Euler sum with a distinct MZV.

\section{A Watson Integral}

Evaluate

$$
W=\frac{1}{\pi^{3}} \int_{0}^{\pi} \int_{0}^{\pi} \int_{0}^{\pi} \frac{1}{3-\cos (x)-\cos (y)-\cos (z)} d x d y d z .
$$

\section{History and Context}

The integral arises in Gaussian and spherical models of ferromagnetism and in the theory of random walks and leads to one of the most impressive closed-form evaluations of an equivalent multiple integral due to G.N. Watson:

$$
\begin{aligned}
\widehat{W} & =\int_{-\pi}^{\pi} \int_{-\pi}^{\pi} \int_{-\pi}^{\pi} \frac{1}{3-\cos (x)-\cos (y)-\cos (z)} d x d y d z \\
& =\frac{1}{96}(\sqrt{3}-1) \Gamma^{2}\left(\frac{1}{24}\right) \Gamma^{2}\left(\frac{11}{24}\right) \\
& =4 \pi(18+12 \sqrt{2}-10 \sqrt{3}-7 \sqrt{6}) \mathrm{K}^{2}\left(k_{6}\right),
\end{aligned}
$$

where $k_{6}=(2-\sqrt{3})(\sqrt{3}-\sqrt{2})$ is the sixth singular value. The most self contained derivation of this very subtle result is due to Joyce and Zucker [27, 28], where more background can also be found. 


\section{Solution}

In [30], it is shown that a simplification can be obtained by applying the formula

$$
\frac{1}{\lambda}=\int_{0}^{\infty} e^{-\lambda t} d t, \quad \operatorname{Re}(\lambda)>0
$$

to $W_{3}$. The 3 -dimension integral is then reducible to a single integral by using the identity

$$
\frac{1}{\pi} \int_{0}^{\infty} \exp (t \cos \theta) d \theta=I_{0}(t)
$$

taking $I_{0}(t)$ to be the modified Bessel function of the first kind. It follows from this that $W=\int_{0}^{\infty} \exp (-3 t) I_{0}^{3}(t) d t$. This integral can be evaluated to 100 digits in Maple giving

$$
\begin{aligned}
W_{3}= & 0.50546201971732600605200405322714025998512901481742089 \\
& 21889934878860287734511738168005372470698960380 \ldots
\end{aligned}
$$

Finally an integer relation hunt to express $\log W$ in terms of $\log \pi, \log 2, \log \Gamma(k / 24)$ and $\log (\sqrt{3}-1)$ will produce $(74)$.

We may also write $W_{3}$ only as a product of $\Gamma$-values. This is what our Mathematician's ToolKit returned:

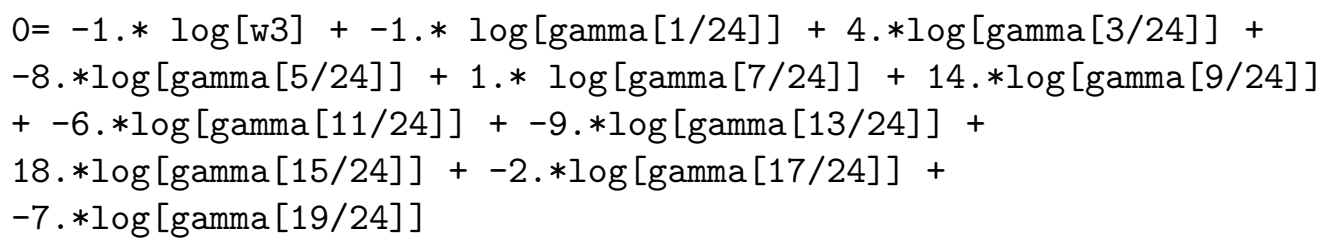

Proving this is achieved by comparing the result with (74) and establishing the implicit $\Gamma$-representation of $(\sqrt{3}-1)^{2} / 96$.

Similar searches suggest there is no similar four dimensional closed form-the Bessel integral being $W_{4}:=\int_{0}^{\infty} \exp (-4 t) I_{0}^{4}(t) d t .\left[\int_{0}^{\infty} \exp (-2 t) I_{0}^{2}(t) d t=\infty\right.$.] In this case it is necessary to compute $\exp (-t) I_{0}(t)$ carefully, using a combination of the formula

$$
\exp (-t) I_{0}(t)=\exp (-t) \sum_{n=0}^{\infty} \frac{t^{2 n}}{2^{2 n}(n !)^{2}}
$$

for $t$ up to roughly $1.2 \cdot d$, where $d$ is the number of significant digits desired for the result, and

$$
\exp (-t) I_{0}(t)=\frac{1}{\sqrt{2 \pi t}} \sum_{n=0}^{N} \frac{\left(\prod_{k=1}^{n} 2 k-1\right)^{2}}{(8 t)^{n} n !}
$$

for larger $t$, where the limit $N$ of the second summation is chosen to be the first index $n$ such that the summand is less than $10^{-d}$ (since this is an asymptotic expansion, taking more terms than $N$ may increase, not decrease the error). We have implemented this as 'besselexp' in our Mathematician's ToolKit, available at http://crd.lbl.gov/ dhbailey/mpdist. Using this software, which includes a PSLQ facility, we found that $W_{4}$ is not expressible as a product of powers of $\Gamma(k / 120)$ (for $0<k<120$ ) with coefficients of less than 12 digits. This result does not, of course, rule out the possibility of a larger relation, but it does cast some doubt, in an experimental sense, that such a relation exists. 


\section{Conclusion}

While all of the problems described herein were studied with a great deal of experimental computation, clean proofs are known for the final results given (except for Problem 7), and in most cases a lot more has by now been proven. Nonetheless, in each case the underlying object suggests plausible generalizations which are still open.

The "hybrid computations" involved in these solutions are quite typical of modern experimental mathematics. Numerical computations by themselves produce no insight, and symbolic computations frequently fail to produce full-fledged, closed-form solutions. But when used together, with significant human interaction, they often are successful in discovering new facts of mathematics and in suggesting routes to formal proof. 


\section{References}

[1] Milton Abramowitz and Irene A. Stegun, Handbook of mathematical functions, Dover Publications, New York, 1970.

[2] Kendall E. Atkinson, An introduction to numerical analysis, Jon Wiley and Sons, New York, 1989.

[3] David H. Bailey, Integer relation detection, Computing in Science and Engineering (2000), $24-28$.

[4] David H. Bailey and Jonathan M. Borwein, Sample problems of experimental mathematics, http://www. expmath.info/expmath-probs.pdf (2003).

[5] David H. Bailey, Jonathan M. Borwein, and Richard E. Crandall, On the Khintchine constant, Mathematics of Computation 66 (1997), no. 217, 417-431.

[6] David H. Bailey, Peter B. Borwein, and Simon Plouffe, On the rapid computation of various polylogarithmic constants, Mathematics of Computation 66 (1997), no. 218, 903-913.

[7] David H. Bailey and David J. Broadhurst, Parallel integer relation detection: Techniques and applications, Mathematics of Computation 70 (2000), no. 236, 1719-1736.

[8] David H. Bailey and Richard E. Crandall, Random generators and normal numbers, Experimental Mathematics 10 (2001), no. 2, 175-190.

[9] , Random generators and normal numbers, Experimental Mathematics 11 (2004), no. $4,527-546$.

[10] David H. Bailey and Xiaoye S. Li, A comparison of three high-precision quadrature schemes, http://crd.lbl.gov/ dhbailey/dhbpapers/quadrature.pdf (2004).

[11] David H. Bailey and Sinai Robins, Highly parallel, high-precision numerical quadrature, http://crd.lbl.gov/ ${ }^{\sim}$ dhbailey/dhbpapers/quadparallel.pdf (2004).

[12] Folker Bornemann, Dirk Laurie, Stan Wagon, and Jörg Waldvogel, the SIAM 100 Digit Challenge: A study in high-accuracy numerical computing, SIAM, Philadelphia, 2004.

[13] J. M. Borwein and D. J. Broadhurst, Determinations of rational Dedekind-zeta invariants of hyperbolic manifolds and feynman knots and links, hep-th/9811173 (1998).

[14] Jonathan Borwein and David Bailey, Mathematics by experiment, A K Peters Ltd., Natick, MA, 2004. MR 2033012

[15] Jonathan Borwein, David Bailey, and Roland Girgensohn, Experimentation in mathematics: Computational paths to discovery, A K Peters Ltd., Natick, MA, 2004.

[16] Jonathan Borwein and Richard Crandall, On the Ramanujan AGM fraction. Part II: the complex-parameter case, Experimental Mathematics (2004).

[17] Jonathan Borwein, Richard Crandall, and Greg Fee, On the Ramanujan AGM fraction. Part I: the real-parameter case, Experimental Mathematics (2004). 
[18] Jonathan M. Borwein and Peter B. Borwein, Pi and the AGM, Canadian Mathematical Society Series of Monographs and Advanced Texts, 4, John Wiley \& Sons Inc., New York, 1998. MR 99h:11147

[19] Jonathan M. Borwein, David M. Bradley, David J. Broadhurst, and Petr Lisoněk, Special values of multiple polylogarithms, Trans. Amer. Math. Soc. 353 (2001), no. 3, 907-941. MR 2003i:33003

[20] Peter Borwein, An efficient algorithm for the Riemann zeta function, http://www.cecm.sfu.ca/personal/pborwein/PAPERS/P155.pdf (1995).

[21] Richard E. Crandall, Topics in advanced scientific computation, Springer-Verlag, New York, 1996. MR 97g:65005

[22] _ New representations for the Madelung constant, Experiment. Math. 8 (1999), no. 4, 367-379. MR 2000m:11125

[23] Richard E. Crandall and Joseph P. Buhler, Elementary function expansions for Madelung constants, J. Phys. A 20 (1987), no. 16, 5497-5510. MR 88m:82034

[24] Helaman R. P. Ferguson, David H. Bailey, and Stephen Arno, Analysis of PSLQ, an integer relation finding algorithm, Mathematics of Computation 68 (1999), no. 225, 351-369.

[25] James Gleick, Chaos: The making of a new science, Penguin Books, New York, 1987.

[26] Julian Havel, Gamma: Exploring Euler's constant, Princeton University Press, 2003.

[27] G. S. Joyce and I. J. Zucker, Evaluation of the Watson integral and associated logarithmic integral for the d-dimensional hypercubic lattice, J. Phys. A 34 (2001), no. 36, 7349-7354. MR 2002i:33018

[28] _ On the evaluation of generalized Watson integrals, Proceedings of the AMS (to appear).

[29] I. Kotsireas and K. Karamanos, Exact computation of the bifurcation point $B_{4}$ of the logistic map and the Bailey-Broadhurst conjectures, International Journal of Bifurcation and Chaos 14 (2004), no. 7, 2417-2423.

[30] A.A. Maradudin, E. W. Montroll, G. H. Weiss, Robert Herman, and H. W. Milnes, Green's functions for monatomic simple cubic lattices, Acad. Roy. Belg. Cl. Sci. Mém. Coll. in-4 deg. (2) 14 (1960), no. 7, 176. MR 22 \#7440

[31] Daniel Shanks and J. W. Wrench Jr., Khintchine's constant, Amer. Math. Monthly 66 (1959), 276-279. MR 21 \#1950

[32] Steven H. Strogatz, Nonlinear dynamics and chaos: With applications to physics, biology, chemistry and engineering, Perseus Book Group, 2001.

[33] Andrew Vaught, personal communication, (2004).

[34] Steven Wolfram, A new kind of science, Wolfram Research, 2002. 